\title{
A TOPOLOGICAL PROOF OF A THEOREM OF KNESER
}

\author{
BJORN FRIBERG ${ }^{1}$
}

\begin{abstract}
We give an elementary topological proof that the orthogonal groups $O(2)$ and $O(3)$ are strong deformation retracts of the space of homeomorphisms (with the compact-open topology) of $R^{2}$ and $S^{2}$, respectively. We also deform the space of bounded homeomorphisms of $R^{2}$ to $S^{1}$.
\end{abstract}

A well-known theorem of Kneser [6] says that the orthogonal groups $O(2)$ and $O(3)$ are strong deformation retracts of the space of homeomorphisms (with the compact-open topology) of $R^{2}$ and $S^{2}$, respectively. His proof, in the setting of complex analytic function theory, is unelementary and untopological and therefore not accessible to many topologists.

The main purpose of this paper is to give an elementary topological proof of his theorem. Thus we prove:

THEOREM 1. The rotation group $S O(2)$ is a strong deformation retract of the space $\mathrm{H}^{+}\left(R^{2}\right)$ of orientation preserving homeomorphisms of the plane.

Corollary 2. $O(2)$ is a strong deformation retract of $H\left(R^{2}\right)$.

COROllary 3. $O(3)$ is a strong deformation retract of $H\left(S^{2}\right)$.

We also obtain the following:

COROLlary 4. The space $B H\left(R^{2}\right)$ of bounded homeomorphisms of the plane deforms to $S^{\mathbf{1}}$.

The methods used in this paper are also desirable as they function well in higher dimensions.

1. Definitions and preliminaries. Let $R^{2}, S^{1}$, and $S^{2}$ denote Euclidean 2 space, the unit spheres in Euclidean 2 and 3 space, respectively. Let $B_{r}$ denote the ball of radius $r$ in $R^{2} . H(X)$ will denote the space of homeomorphisms of $X$, with the compact-open topology. $H^{+}(X)$ will denote

Presented to the Society, June 19, 1971; received by the editors May 8, 1972.

AMS (MOS) subject classifications (1970). Primary 57E05, 57A05, 58D05.

Key words and phrases. Homeomorphism, deformation, isotopy, topological group, bounded homeomorphism.

${ }^{1}$ This work represents part of the author's dissertation at the University of California, Los Angeles, under the direction of D. S. Gillman and R. C. Kirby.

(C) American Mathematical Society 1973 
those homeomorphisms which preserve orientation. A basis for the neighborhoods of the identity $=$ id consists of sets of the form $N(C, \varepsilon)=$ $\{h|h(x)-x|<\varepsilon$ all $x \in C\}$, where $C$ is compact and $|\cdot|$ denotes the usual norm. In this paper $X$ will be a subset of $R^{2}$ or $S^{2}$, and we will usually just write $H$ in place of $H(X)$ when it is clear what $X$ we are regarding. By [1], [2], $H$ will then be a topological transformation group on $X$ and compositions, inverses, and evaluations will be continuous. If $C$ is a compact subset of $R^{2}, h \in H\left(R^{2}\right)$, and $x \in h(C)$, then by $\operatorname{rad}_{x}\{h(C)\}$ $\left(\operatorname{Rad}^{x}\{h(C)\}\right)$ we will mean the radius of the largest (smallest) ball centered at $x$ which is contained in (contains) $h(C)$. If $x=$ origin, it is usually omitted.

An isotopy $h_{t}$ of $h \in H$ is a path in $H$ starting at $h$. We say an isotopy $h_{t}$ for each $h \in G \subset H$ is canonical if the function from $G \times I$ into $H$ defined by $(h, t) \rightarrow h_{t}$ is continuous, i.e., defines a deformation of $G$ in $H$.

If $0 \leqq t \leqq 1$ and $a<b, c<d$, by $T_{t}(a ; b ; c ; d)$ we mean the homeomorphism of $R^{2}$ which is fixed on $B_{a}$ and off $B_{d}$ and takes a ray emanating from the origin onto itself as follows. $T_{t}$ is fixed on $[0, a]$ and on $[d, \infty)$, and takes $b$ to $(1-t) b+t c$ and is linear on $[a, b]$ and $[b, d] . T_{t}(a ; b ; c ; d)$ is easily seen to be continuous viewed as a function from a subset of $R^{5}$ into $H\left(R^{2}\right)$.

2. Radial squeezing. The following lemma is well known. Our version varies slightly in that the orthogonal group is to remain invariant. For ease of notation we regard $R^{2}$ as $[-\infty, \infty) \times S^{1}$ with $\{-\infty\} \times S^{1}$ squeezed to the origin. Then by the ball $B_{r}$ of radius $r$, we mean the set $[-\infty, r] \times S^{\mathbf{1}}$.

Lemma 5. Let $h \in H\left(R^{2}\right)$ fix the origin. Then $h$ is canonically isotopic to $h_{\infty}$, with $B_{j-1} \subset h_{\infty}\left(B_{j}\right) \subset B_{j}$ for all $j \in J$. If $h \in O(2)$, then $h_{t}=h$ all $t \in[0, \infty]$.

Proof. By squeezing we can assume that $B_{-1} \subset h\left(B_{0}\right) \subset B_{0}$. Inductively assume that $h$ has been canonically isotoped to $h_{n}$, with $B_{j-1} \subset h_{n}\left(B_{j}\right) \subset B_{j}$ for all $|j| \leqq n$.

Set

$$
\begin{aligned}
a_{n} & =\operatorname{rad} h_{n}^{-1}\left(B_{n-1}\right), \\
b_{n} & =\min \left[n+1 ; \operatorname{rad}\left\{h_{n}^{-1}\left(B_{n+1}\right)\right\}\right], \\
c_{n} & =\min \left[n+1 ; \operatorname{rad}\left\{h_{n}\left(B_{n+1}\right)\right\}\right], \\
\Phi_{n} & =h_{n} \circ T_{1}\left(a_{n}-1 ; n ; a_{n} ; b_{n}\right) \circ h_{n}^{-1}, \\
h_{n+t} & =\Phi_{n}^{-1} \circ T_{4 t}\left(n-1 ; c_{n} ; n+1 ; n+2\right) \circ \Phi_{n} \circ h_{n}, \\
h_{n+1 / 4+t} & =T_{4 t}\left(n ; d_{n} ; n+1 ; d_{n}+1\right) \circ h_{n+1 / 4},
\end{aligned}
$$

where

$$
d_{n}=\operatorname{Rad} h_{n+1 / 4}\left(B_{n+1}\right) \quad \text { for } 0 \leqq t \leqq \frac{1}{4} .
$$


Thus the radial structure of $h_{n}$ gives us the homeomorphism $\Phi_{n}$ which puts $h_{n}\left(B_{n}\right)$ inside $B_{n-1}$, and is fixed off $B_{n+1}$. We then use the radial structure given by $\Phi_{n}$ to slide $h_{n}\left(B_{n+1}\right)$ outside of $B_{n+1}$, leaving $h_{n}\left(B_{n}\right)$ alone. A final squeeze then puts $h_{n}\left(B_{n+1}\right)$ where it belongs. We next perform the analogous isotopy to $h_{n+1 / 2}$ near $B_{-n}$ to get $h_{n+1}$, with

$$
B_{j-1} \subset h_{n+1}\left(B_{j}\right) \subset B_{j} \text { for }|j| \leqq n+1 \text {. }
$$

One easily checks that limit ${ }_{n \rightarrow \infty} h_{n}=h_{\infty}$ exists, and has the desired properties. That the isotopy is canonical follows from the continuity of the $a_{n}, b_{n}, c_{n}, d_{n}$.

3. Bounded homeomorphisms. We say that $h$ is bounded by $M$ if $|h(x)-x| \leqq M$ for all $x \in R^{2}$. We denote by ${ }^{M} B H\left(R^{2}\right)$ the collection of all homeomorphisms bounded by $M$, and by $B H\left(R^{2}\right)=\bigcup_{M}{ }^{M} B H\left(R^{2}\right)$, the group of all bounded homeomorphisms. If $h$ is bounded, the Alexander isotopy gives an isotopy of $h$ to the identity; namely

$$
\begin{aligned}
h_{t} & =\phi_{t} \circ h \circ \phi_{t}^{-1}, & & 0 \leqq t<1, \\
& =\mathrm{id}, & t & =1,
\end{aligned}
$$

where $\phi_{t} \in H\left(R^{2}\right)$ is defined by $\phi_{t}(x)=(1-t) x$. However the Alexander isotopy is not canonical. We do have the following:

LEMMA 6. The Alexander isotopy is canonical when restricted to ${ }^{M} B H\left(R^{2}\right)$.

Proof. Given $h$ and $t=1$ with $N\left(B_{k}, \varepsilon\right)$ a neighborhood of $h_{1}=\mathrm{id}$. Choose $t_{0}>1-\varepsilon / M$. Then for $t>t_{0}$ and any $h \in{ }^{M} B H\left(R^{2}\right)$, we have that $h_{t}$ is bounded by $\varepsilon$, i.e., $h_{t} \in N\left(B_{k}, \varepsilon\right)$.

LEMMA 7. Let $h \in H^{+}\left(R^{2}\right)$ be bounded by $M$ on the first factor, i.e., $\left|\Pi_{1} h(x, y)-x\right| \leqq M$ for all $(x, y) \in R^{2}$. Then $h$ is canonically isotopic to the identity. (Here $\Pi_{i}, i=1,2$, means projection on the ith factor.)

Proof. Given $h$, we define a sequence of functions $f_{j}: R \rightarrow R, j \in J$, such that

(i) $f_{j}<f_{j+1}$,

(ii) $G_{2 j-1}<h\left(G_{2 j}\right)<G_{2 j+1}$

where $G_{j}$ is the graph of $f_{j}$, i.e., the set $\left\{\left(x, f_{j}(x)\right) \mid x \in R\right\}$ and " $<$ " in (ii) means lies below in the usual sense.

Set

$$
\begin{aligned}
f_{0} & =0, \\
f_{1}\left(x_{0}\right) & =\max \left[1 ; \sup \left\{\Pi_{2} \circ h(x, 0)|| x-x_{0} \mid \leqq 2 M\right\}\right], \\
f_{-1}\left(x_{0}\right) & =\min \left[-1 ; \inf \left\{\Pi_{2} \circ h(x, 0)|| x-x_{0} \mid \leqq 2 M\right\}\right], \\
f_{2}\left(x_{0}\right) & =\max \left[1+f_{1}\left(x_{0}\right) ; \sup \left\{\Pi_{2} \circ h^{-1}\left(x, f_{1}(x)\right)|| x-x_{0} \mid \leqq 2 M\right\}\right], \\
f_{-2}\left(x_{0}\right) & =\min \left[-1+f_{-1}\left(x_{0}\right) ; \inf \left\{\Pi_{2} \circ h^{-1}\left(x, f_{-1}(x)\right)|| x-x_{0} \mid \leqq 2 M\right\}\right] .
\end{aligned}
$$


For $j \geqq 1, f_{j+2}\left(f_{-(j+2)}\right)$ is defined inductively with $f_{j+1}\left(f_{-(j+1)}\right)$ in place of $f_{j-1}\left(f_{-(j-1)}\right)$ in the formula for $f_{j}\left(f_{-j}\right)$.

Define $\Phi_{1}(h) \in H^{+}\left(R^{2}\right)$ to be the vertical homeomorphism, which on each fiber $\{x\} \times R$ takes $(x, j)$ to $\left(x, f_{j}(x)\right)$ and is linear on the segments $[j, j+1]$, for all $j \in J . \Phi_{1}(h)$ is isotopic to the identity via $\Phi_{t}(h)$ in the natural way.

Set $h_{t}=\Phi_{t}(h)^{-1} \circ h \circ \Phi_{t}(h)$. If $R_{j}=\{(x, j) \mid x \in R\}$, then from (ii) it follows that $R_{2 j-1}<h_{1}\left(R_{2 j}\right)<R_{2 j+1}$, i.e. $\left|\mathrm{II}_{2} \circ h_{1}(x, y)-y\right| \leqq 2$ while

is still bounded by $M$.

$$
\left|\Pi_{1} \circ h_{1}(x, y)-x\right|
$$

One easily checks that $h \rightarrow \Phi_{t}(h)$ is continuous and that the isotopy is canonical. The result now follows from Lemma 6.

4. Lifting homeomorphisms. We consider the following covering map $\lambda: R^{2} \rightarrow R \times S$, defined as follows. Let $e: R \rightarrow S$ be the exponential covering map given by $e(r)=e^{\Pi i r}$. We take $\lambda$ to be an approximation to id $\times e$ which equals id $\times e$ off a compact neighborhood of the point $(\mathrm{id} \times e)(1,0)=(1,0)$ and which is the identity on a smaller compact neighborhood $C$ of $(1,0)$. Thus in "wrapping" $R^{2}=R_{+} \times R$ around $R_{+} \times S=R \times S$, we merely place our finger over the point $(1,0)$ which fixes $C$.

LEMMA 8. Let $\lambda$ be as above. Let $h \in H\left(R^{2}\right)$ fix the origin and $(1,0)$. Then $\left.h\right|_{R^{2-0}} \in H(R \times S)$ lifts (via $\left.\lambda\right)$ uniquely to $\hat{h} \in H\left(R^{2}\right)$ fixing $(1,0)$. Furthermore $h$ is canonically isotopic to $\hat{h}$.

Proof. The existence and uniqueness of $\hat{h}$ follows from $\mathrm{Hu}[4$, p. 90]. Clearly $h=\hat{h}$ on $h^{-1}(C) \cap C$. We define

$$
\begin{aligned}
h_{t} & =\tau \circ \phi_{t}^{-1} \circ \tau^{-1} \circ \hat{h}^{-1} \circ h \circ \tau \circ \phi_{t} \circ \tau^{-1} \quad \text { for } 0 \leqq t<1, \\
& =\hat{h} \quad \text { for } t=1,
\end{aligned}
$$

where $\tau$ is translation by the point $(1,0)$ and $\phi_{t}(x)=(1-t) x$.

Since $\tau^{-1} \circ \hat{h}^{-1} \circ h \circ \tau=\mathrm{id}$ on a neighborhood of the origin, $h_{t}$ is clearly an isotopy. That it is a canonical isotopy follows from the continuity of the functions $h \rightarrow \hat{h}$ and $h \rightarrow \operatorname{rad}_{(1,0)}\left\{C \cap h^{-1}(C)\right\}$, which can easily be established by standard $\delta-\varepsilon$ arguments.

5. Proof of Theorem 1. Let $h \in H^{+}\left(R^{2}\right)$. We will give a canonical isotopy $h_{t}, 0 \leqq t \leqq 4$, so that $h_{4} \in S O(2)$ is a strict rotation. Furthermore if $h \in S O(2)$, then $h_{t}=h$ all $t$.

Step (1). By canonically translating along the line segment determined by $h(0)$ and 0 , we get $h_{1}$ fixing the origin.

Step (2). We now apply Lemma 5 to $h_{1}$ to get $h_{2}$ which is small on the radial factor, as in the conclusion of Lemma 5 .

Step (3). Consider $h_{2}(1,0)=\left(r, \theta_{h}\right)$, written in polar coordinates. By canonically translating in a coordinate neighborhood of $\left(1, \theta_{h}\right)$ we get $h_{3}$, 
with $h_{3}(1,0)=\left(1, \theta_{h}\right)$ in polar coordinates. Let $\rho_{h} \in S O(2)$ be rotation by $\theta_{h}$. Set $g=\rho_{h}^{-1} \circ h_{3}$. Then $g$ fixes the origin and $(1,0)$. We canonically isotope $h_{3}$ to $\rho_{h}$ by canonically isotoping $g$ to the identity.

Step (4). $g$ satisfies the hypothesis of Lemma 8. Furthermore $\hat{g}$ will be bounded on the first $R$ factor by 2 since $h_{3}$ (hence $g$ ) was so bounded radially. Thus $\hat{g}$ satisfies the hypothesis of Lemma 7 . This completes the canonical isotopy of $g$ to the identity, and of $h_{3}$ to $\rho_{h}=h_{4}$.

One easily checks that if $h \in S O(2)$, then $h_{t}=h$ all $t$. In Step (4), $g$ will then be the identity, and so will be the isotopy from $g$ to the identity. (Note. in Step (4), $\hat{g}$ may move the origin, hence the isotopy from $h_{3}$ to $h_{4}$ will also. Thus, if we wish, we could translate this part of the isotopy, which would then fix the origin.)

\section{Proof of the corollaries.}

Proof of Corollary 2. Just apply the analogous isotopy to the other component.

Proof of Corollary 3. Let $p, q$, and $r$ correspond to the origin, $\infty$, and $(1,0)$ under stereographic projection. Let $h \in H\left(S^{2}\right)$ be given.

Step 1. If $h(p)$ and $h(q)$ are antipodal, leave $h$ alone. Otherwise, slide $h(p)$ and $h(q)$ along the geodesic they form until they are antipodal. More precisely, we define $\phi_{t}(x, y) \in H\left(S^{1}\right), x, y \in S^{1}$, by $\phi_{t}(x, y)=$ id if $x$ and $y$ are antipodal. If not, let $a$ and $b$ be the midpoints of the shorter and longer arcs from $x$ to $y$. Let $c$ and $d$ be the midpoints of the arcs from $a$ to $b$, containing $x$ and $y$ respectively. Then $\phi_{t}(x, y)$ is the linear homeomorphism of $S^{1}$ determined by $a \rightarrow a ; b \rightarrow b ; x \rightarrow(1-t) x+t c ; y \rightarrow(1-t) y+t d$. Consider $S^{2}$ as $S^{1} \times[-1,+1]$ with each end squeezed to a point. Set $\psi_{t}(x, y)=\phi_{t}(x, y) \times \mathrm{id} \in H\left(S^{2}\right)$. Thus we set $h_{t}=\psi_{t}(h(p), h(q)) \circ h . h_{t}$ is clearly canonical since if $h(p)$ and $h(q)$ are almost antipodal, the isotopy is small. In much the same manner we slide $h_{1}(r)$ to the midpoint of the arc through $h_{1}(p), h_{1}(q)$, and $h_{1}(r)$, getting $h_{2}$ which then uniquely determines $\theta \in O(3)$. Setting $g=\theta^{-1} \circ h_{2}$ fixes $p, q$, and $r$.

Step 2. Consider $\left.g\right|_{S^{2}-\{\alpha\}} \in H^{+}\left(R^{2}\right)$ fixing the origin and $(1,0)$. As in the proof of Theorem $1, g$ is is canonically isotopic to the identity. We extend the isotopy to $S^{2}$ and thus get that $h_{2}$ (and $h$ ) is canonically isotopic to $\theta$.

Proof of Corollary 4. Regard $S^{1}=[0,1]$ with the endpoints identified. Define an embedding $i: S^{1} \rightarrow B H\left(R^{2}\right)$ by (for $0 \leqq t<1$ )

$$
\begin{aligned}
& \begin{aligned}
i(t) & =\text { rotation by } 2 \pi t \text { on } B_{1 /(1-t)}, \\
& =\text { rotation by }\{2-r(1-t)\} 2 \pi t \text { on } S_{r}, \quad \frac{1}{1-t} \leqq r \leqq \frac{2}{1-t}, \\
& =\mathrm{id} \text { off } B_{2 /(1-t)}
\end{aligned} \\
& \text { for } t=1, i(t)=\mathrm{id} .
\end{aligned}
$$


Since limit thi $i(t)=\mathrm{id}, i$ is clearly an embedding. Let $h \in B H\left(R^{2}\right)$. We canonically isotope $h$ into $i\left(S^{1}\right)$ by replacing $S O(2)$ by $i\left(S^{1}\right)$ in the proof of Theorem 1 and essentially performing the isotopy given by Theorem 1 . However $h_{t}$ might not be bounded, so we modify the isotopy slightly. In particular, applying Steps 1 and 3 we get $h_{2}$ fixing the origin with $h_{2}(1,0)=\left(1, \theta_{h}\right)$ in polar coordinates. Set $g=\rho^{-1} \circ h_{2}$, where $\rho \in i\left(S^{1}\right)$ is uniquely determined by $\theta_{h}$. Then $g$ is bounded and fixes the origin and $(1,0)$. We isotope $g$ to the identity through bounded homeomorphisms as follows. We alter the isotopy of Lemma 5 and the first part of the isotopy of Lemma 7 to be fixed on a neighborhood of $(1,0)$. Then performing Steps 2 and 4 to $g$ gives us $\bar{g} \in{ }^{M} B H$, with $g=\bar{g}$ on a neighborhood $N_{g}$ of $(1,0)$ with $g \rightarrow \operatorname{rad}_{(1,0)}\left(N_{g}\right)$ continuous. Then $g$ is canonically isotopic to $\bar{g}$ through bounded homeomorphisms by the isotopy of Lemma 8 , while $\bar{g}$ is canonically isotopic to the identity through bounded homeomorphisms (by the Alexander isotopy).

\section{REFERENCES}

1. Richard F. Arens, A topology for spaces of transformations, Ann. of Math. (2) 47 (1946), 480-495. MR 8, 165.

2. _ Topologies for homeomorphism groups, Amer. J. Math. 68 (1946), 593610. MR 8, 479.

3. Bjorn Friberg, Ph.D. Thesis, University of California, Los Angeles, Calif., 1971.

4. Sze-Tsen Hu, Homotopy theory, Pure and Appl. Math., vol. 8, Academic Press, New York, 1959, MR 21 \#5186.

5. J. M. Kister, Micro bundles are fiber bundles, Ann. of Math. (2) 80 (1964), 190199. MR 31 \#5216.

6. Hellmuth Kneser, Die Deformationssatze der einfach zusammenhangenden Flachen, Math. Z. 25 (1926), 362-372.

Department of Mathematics, University of California at los Angeles, Los ANGeles, California 90024

Department of Mathematics, University of British Columbia, Vancouver 8, B.C., CANADa

Current address: Department of Mathematics, University of Saskatchewan, Saskatoon, Saskatchewan, Canada 